\title{
Effects of Community Mental Health Centers on Oxidative Stress in Patients with Schizophrenia
}

\author{
Burak Kulaksizoglu ${ }^{*}$, Sibel Kulaksizoglu ${ }^{2}$ \\ ${ }^{1}$ Psychiatry Department, Antalya Education and Research Hospital, Antalya, Turkey \\ ${ }^{2}$ Biochemistry Department, Antalya Education and Research Hospital, Antalya, Turkey \\ Email: `burakulaksiz@yahoo.com, sblclngrgl@yahoo.co.uk
}

How to cite this paper: Kulaksizoglu, B. and Kulaksizoglu, S. (2018) Effects of Community Mental Health Centers on Oxidative Stress in Patients with Schizophrenia. International Journal of Clinical Medicine, 9, 423-431. https://doi.org/10.4236/ijcm.2018.95036

Received: March 13, 2018

Accepted: May 15, 2018

Published: May 18, 2018

Copyright ( 92018 by authors and Scientific Research Publishing Inc. This work is licensed under the Creative Commons Attribution International License (CC BY 4.0).

http://creativecommons.org/licenses/by/4.0/

(c) (i) Open Access

\begin{abstract}
Objective: The aim of our study was to evaluate of oxidative stress between the schizophrenic patients who regularly continued to Community Mental Health Centers (CMHC) and the patients who did not continue to CMHC. By this study, the effects of CMHC on oxidative stress between these two group of patients were assessed. Methods: Total number of 86 volunteers were enrolled in this study; 43 (27 males, 16 females) patients who regularly continued (average 2 years, at least 3 days a week) to CMHC and 43 (25 males, 18 females) patients who regularly did not continue to CMHC. Total antioxidative stress (TAS), total oxidative stress (TOS), oxidative stress index (OSI), paraoxanase (PON1), arylesterase (ARE) and total thiol (T.Thl) levels were measured with a novel automated method. Positive and Negative Syndrome Scale (PANSS) was used to assess the patients. Results: TOS, PANSS-Negative subscale and PANSS-Total subscale were found to be significantly higher in the group of patients who were not continuing to CMHC, than the group of patients who were continuing to CMHC. ARE and T.thl were found significantly lower in the group of patients who were not continuing to CMHC. There was a significant positive correlation between PANSS-Negative Subscale and TOS, OSI in the group of patients who were not continuing to CMHC. There was a positive correlation between PANSS-Negative Subscale and TOS in the group of patients who were continuing to CMHC. Conclusions: Oxidative stress and negative symptoms of schizophrenia in the group of patients who were continuing to CMHC were less than in the group of patients who were not continuing to CMHC. Thus, regular follow up at $\mathrm{CMHC}$ is very important in the treatment of schizophrenia patients.
\end{abstract}

\section{Keywords}

Community Mental Health Centers, Oxidative Stress, Schizophrenia, PANSS 


\section{Introduction}

Schizophrenia is a kind of disease which has social, economical, legal, and medical dimensions. Not only the patients but also the family and the soceity are affected in different ways. Schizophrenia reduces the quality of life and the life span. At the same time, primary caregivers are affected from economic and psychosocial fronts because of the disease burden and also the family is depressed [1]. In the treatment of schizophrenia, the target is not only to reduce the severity of symtoms but also to increase the quality of life and the productivity [2].

In many scountries, Community Mental Health Centers (CMHC) were established for the rehabilitation of schizophrenic patients. It was shown that CMHC improve the quality of life and functionality of the schizophrenic patients and also reduce the loss of ability [3]. Antalya Education and Research Hospital CMHC started to serve on May 15, 2012. Rehabilitation programs made for the integration of patients into society improve psychosocial skills acquisition and psychological support. On all weekdays, schizophrenic patients are admitted to the center. The various training courses, individual assessments, psychological counseling, group therapies, and treatment follow-up and controls are done in the routine functioning of the center. Furthermore, good morning meetings, painting, wood painting, folk dances, sports, folk music choir works, cooking training, and nature walks are the part of rehabilitation programs which are applied on certain weekdays. In the studies, it was shown that: CMHC decreases the frequency of psychotic attacks and length of stay in hospital in patients with schizophrenia. Social service workers are interested in the economic status of patients in the center, so the economic support is provided to patients who are in low economic situation [4]. As a result, it was shown that, the burden of caregivers' was decreased and positive developments in their lives were seen [5] [6].

One of the important factors in the ethiopathogenesis of schizophrenia is the oxidative stress. Oxidative damage to proteins, and DNA as observed in schizophrenia is known to impair cell function. Currently available evidence shows towards an alteration in the activities of enzymatic and nonenzymatic antioxidant systems in schizophrenia, and oxidative stress induces behavioral and molecular anomalies in schizophrenia [7]. Total antioxidative stress (TAS), total oxidative stress (TOS), oxidative stress index (OSI), paraoxonase (PON1), arylesterase (ARE) and total thiol (T.Thl) are the recently parameters used for determination of the oxidant-antioxidant balance in patients with schizophrenia [8] [9] [10] [11]. Thiol is an organic compound containing sulfhydryl (-SH) group which has a critical role in preventing the occurence of oxidative stress in the cells [12]. PON1 and ARE are esterase derivatives antioxidant enzymes which prevent the peroxidation of the low density lipoproteins especially [13].

There is no data in the literature about the effects of CMHC on oxidative stress in patients with schizophrenia. Regular and moderate exercise has been shown to reduce oxidative stress in studies conducted [14]. However, there is no 
literature on the effects of social activity and exercise on oxidative stress in schizophrenic patients. From this point, our study was the first in this respect. The aim of our study was to evaluate the difference of oxidative stress between the schizophrenic patients who regularly continued to CMHC and who did not continue.

\section{Methods}

Total number of 86 volunteers were recruited for this study; 43 (27 males, 16 females) patients who regularly continued (average 2 years, at least 3 days a week) to CMHC and 43 (25 males, 18 females) patients who did not continue to CMHC, aged between 25 - 55 years old, were participated into study. The schizophrenic patients had been diagnosed according to the diagnostic criteria of Diagnostic and Statistical Manual of Mental Disorders, Fourth Edition, Text Revision and were followed for at least 2 years in the psychiatry clinic of Antalya Education and Research Hospital [15]. Patients who wanted to participate in the study between January and July 2017 were included in the study. Minimum Sample Size Calculation was not performed. Sociodemographic data forms were filled by the participants. Positive and Negative Syndrome Scale (PANSS) developed by Kay et al. [16], which contained 30 items, and a seven-point disease severity assessment scale was administered to the patients. Fourteen of 30 psychiatric parameters belong to the positive $(n=7)$ and negative $(n=7)$ symptoms subscale, and the remaining 16 parameters belong to the general psychopathology subscale. During the follow-up period, the detection of disease symptoms at least once indicated positivity of the relevant symptoms.

Patients were excluded from the study if they met one or more of the following criteria: hypertension, heart disease, diabetes mellitus, hepatic or renal failure, active infection, active or chronic inflammatory diseases, heavy smoking (15 cigarettes per day), obesity (body mass index $30 \mathrm{~kg} / \mathrm{m}^{2}$ ), and treatment with antiinflammatory, malignity, vitamin or antioxidant supplements, or immunosuppressive medications, autoimmune diseases, alcohol and substance abuse or dependence.

Antecubital vein blood was taken after 12 hours of fasting from the participant for laboratory analysis. Vacutainer gel tubes were used. Serum was separated by centrifugation $10 \mathrm{~min}$ at $4000 \mathrm{~g}$ and rapidly serum fractions were stored at $-80^{\circ} \mathrm{C}$ and used to analyze PON1, ARE, TOS, TAS, and T.thl concentrations. TAS, TOS, PON1, ARE and T.thiol activities were measured by using available kits (Relassay ${ }^{\oplus}$, Turkey).

Antioxidants in the serum reduce the dark blue-green colored 2,2'-azino-bis (3-ethylbenzthiazoline-6-sulfonic acid) radical to a colorless reduced form. The antioxidative effect of the serum against the potent free radical reactions initiated by the produced hydroxyl radical is measured [8]

Oxidants present in the serum oxidize the ferrous ion-chelator complex to ferric ion. The ferric ion makes a colored complex, which can be measured 
spectrophotometrically [8].

The OSI value was calculated according to the following formula: OSI (arbitrary unit $)=$ TOS (micromolar hydrogen peroxide equivalent per liter)/TAS (micromolar trolox equivalent per liter) [17].

PON1 activity measurement method consists of two different sequential reagents; the first reagent is Tris buffer and it also contains calcium ion, which is a cofactor of PON1 enzyme. Non-enzymatic hydrolysis of paraoxon was subtracted from the total rate of hydrolysis. PON1, present in the sample, hydrolyses phenylacetate to its products, which are phenol and acetic acid. The phenol is colorimetrically measured via oxidative coupling with 4 -aminoantipyrine and potassium ferricyanide. Phenylacetate was used as a substrate to measure the ARE activity [10] [11].

Serum total thiol concentration or sulfhydryl groups (SH) were measured by the method described by Ellman et al. [18]. and modified by $\mathrm{Hu}$ et al. [19] Briefly, $1 \mathrm{~mL}$ of buffer containing $0.1 \mathrm{M}$ Tris, $10 \mathrm{mM}$ EDTA ( $\mathrm{pH} 8.2$ ), and $50 \mu \mathrm{L}$ serum was added to cuvettes, followed by $50 \mu \mathrm{L}$ of $10 \mathrm{mM}$ DTNB in methanol. Sample absorbance was read at $412 \mathrm{~nm}$ on a spectrophotometer.

\section{Statistical Analysis}

Continuous variables were presented as mean \pm standard deviation, while categorical variables were given as percentages. The Kolmogorov-Smirnov test was used to verify the normality of the distribution of continuous variables. Statistical analysis of clinical data between two groups consisted of unpaired $t$-tests for parametric data and Mann-Whitney $U$-test analysis for nonparametric data. Correlations were assessed with the Pearson/Spearman correlation coefficient. Analyses were performed with PASW 18 (SPSS Inc., Chicago, IL, USA) software and a $P$-value $<0.05$ was considered statistically significant.

\section{Results}

In terms of sociodemographic data, the findings between the groups of patients who continued to CMHC and who did not continue to CMHC were consistent with each other (Table 1). In our study, there was no significant difference at age, gender, and the being used medications between two groups.

TOS, PANSS-Negative subscale and PANSS-Total subscale were found to be significantly higher in the group of patients who were not continuing to CMHC, than the group of patients who were continuing to CMHC. ARE and T.thl were found significantly lower in the group of patients who were not continuing to CMHC. TAS, PON1, OSI and PANSS-Positive subscale values were not different between the two groups (Table 2).

There was a significant positive correlation between PANSS-Negative Subscale and TOS, OSI in the group of patients who were not continuing to CMHC (Table 3).

There was a positive correlation between PANSS-Negative Subscale and TOS in the group of patients who were continuing to CMHC (Table 4). 
Table 1. Sociodemographic characteristics of the patients.

\begin{tabular}{|c|c|c|}
\hline & $\begin{array}{c}\text { Patients who } \\
\text { continued } \\
\text { regularly to CMHC } \\
(\mathrm{n}=43)\end{array}$ & $\begin{array}{l}\text { Patients who did } \\
\text { not continue } \\
\text { regularly to CMHC } \\
(n=43)\end{array}$ \\
\hline Age range (years) & $23-58$ & $22-55$ \\
\hline Age, mean \pm SD & $41.67 \pm 8.90$ & $38.90 \pm 16.89$ \\
\hline \multicolumn{3}{|l|}{ Gender (n, (\%)) } \\
\hline Female & 16. (37.2) & 18. (41.9) \\
\hline Male & 27. (62.8) & 25. (58.1) \\
\hline \multicolumn{3}{|l|}{ Marital status (n, (\%)) } \\
\hline Single & $35(81.39)$ & $37(86)$ \\
\hline Divorced, separate & $5(11.62)$ & $4(9.30)$ \\
\hline Married & $3(6.97)$ & $2(4.65)$ \\
\hline \multicolumn{3}{|l|}{ Education (n, (\%)) } \\
\hline Literate & $9(20.93)$ & $11(25.58)$ \\
\hline Primary school graduate & $10(23.25)$ & $14(32.55)$ \\
\hline Secondary school, high school & $21(48.83)$ & $16(37.21)$ \\
\hline University graduate & $3(6.97)$ & $2(4.65)$ \\
\hline Duration of disease (year) (mean \pm SD) & $12 \pm 1.22$ & $13 \pm 27$ \\
\hline \multicolumn{3}{|l|}{ Drug therapy $(\mathrm{n},(\%))$} \\
\hline Atypical antipsychotic & $31(72.09)$ & $30(69.76)$ \\
\hline Typical antipsychotic & $3(6.97)$ & $2(4.65)$ \\
\hline Typical/atypical antipsychotic & $9(20.93)$ & $11(25.58)$ \\
\hline \multicolumn{3}{|l|}{ Type of schizophrenia (n, (\%)) } \\
\hline Paranoid & $36(83.72)$ & $33(76.74)$ \\
\hline Disorganized & $0(0)$ & $5(11.62)$ \\
\hline Undifferentiated & $3(6.97)$ & $3(6.97)$ \\
\hline Residual & $4(9.30)$ & $2(4.65)$ \\
\hline
\end{tabular}

Notes: ${ }^{\star}$ Correlation is significant at the 0.05 level. Abbreviations: SD, Standard deviation.

Table 2. Comparison of TAS, TOS, OSI, ARE, PON1, and T.thl values between the groups of patients who continued to CMHC and who did not continue to CMHC.

\begin{tabular}{cccc}
\hline & $\begin{array}{c}\text { Patients } \\
\text { Continued } \\
\text { regularly to CMHC } \\
(\mathbf{n}=43) \\
\text { Mean } \pm \mathrm{SD}\end{array}$ & $\begin{array}{c}\text { Patients did not } \\
\text { Continue } \\
\text { regularly to CMHC } \\
(\mathbf{n}=43) \\
\text { Mean } \pm \mathrm{SD}\end{array}$ & $\mathrm{p}$ \\
\hline TAS $(\mathrm{nmol}$ trloksl) & $2.84 \pm 0.62$ & $3.06 \pm 0.48$ & 0.075 \\
TOS $\left(\mu \mathrm{mol} \mathrm{H}_{2} \mathrm{O}_{2}\right.$ Equiv./L) & $15.83 \pm 2.76$ & $18.09 \pm 3.28$ & $<0.001^{*}$ \\
OSI & $5.93 \pm 1.97$ & $6.07 \pm 1.58$ & 0.704 \\
\hline
\end{tabular}




\section{Continued}

\begin{tabular}{cccc}
\hline ARE $(\mathrm{kU} / \mathrm{L})$ & $40.63 \pm 15.30$ & $33.51 \pm 15.28$ & $0.034^{*}$ \\
PON1(U/L) & $34.19 \pm 2.10$ & $33.37 \pm 5.07$ & 0.326 \\
T.thl $(\mathrm{mm} / \mathrm{L})$ & $533.69 \pm 245.31$ & $438.57 \pm 109.13$ & $0.024^{*}$ \\
PANSS-Negative Subscale & $14.54 \pm 5.76$ & $16.74 \pm 3.44$ & $0.035^{*}$ \\
PANSS-Positive Subscale & $15.05 \pm 3.21$ & $15.56 \pm 4.66$ & 0.555 \\
PANSS-Total Subscale & $61.21 \pm 8.14$ & $64.72 \pm 7.92$ & $0.046^{*}$ \\
\hline
\end{tabular}

${ }^{*}$ Correlation is significant at the 0.05 level (2-tailed). Mann-Whitney U-test and Student's t-test were used. TAS, total antioxidant status; TOS, total oxidative status; OSI, oxidative stress index; PANSS, Positive and Negative Syndrome Scale; PON1, paraoxonase; ARE, arylesterase; T.thl, total thiol.

Table 3. Relationship between PANSS and TAS, TOS, OSI, ARE, PON1, and T.thiol values in the group of patients who did not continue to CMHC.

\begin{tabular}{ccccccc}
\hline & \multicolumn{2}{c}{ PANSS-Negative Subscale } & \multicolumn{2}{c}{ PANSS-Positive Subscale } & \multicolumn{2}{c}{ PANSS-Total Subscale } \\
\cline { 2 - 7 } & $\mathrm{R}$ & $\mathrm{P}$ & $\mathrm{R}$ & $\mathrm{P}$ & $\mathrm{R}$ & $\mathrm{P}$ \\
\hline TAS & -0.136 & 0.383 & -0.050 & 0.752 & -0.117 & 0.454 \\
TOS & 0.310 & $0.043^{*}$ & -0.112 & 0.476 & 0.188 & 0.228 \\
OSI & 0.304 & $0.047^{*}$ & -0.018 & 0.909 & 0.240 & 0.122 \\
ARE & 0.151 & 0.333 & -0.111 & 0.477 & 0.011 & 0.943 \\
PON1 & 0.051 & 0.746 & 0.127 & 0.416 & 0.141 & 0.367 \\
T.thl & 0.135 & 0.386 & -0.096 & 0.539 & 0.008 & 0.957 \\
\hline
\end{tabular}

*Correlation is significant at the 0.05 level (2-tailed). Pearson and Spearman correlation analyses were used. TAS, total antioxidant status; TOS, total oxidative status; OSI, oxidative stress index; PANSS, Positive and Negative Syndrome Scale; PON1, paraoxonase; ARE, arylesterase; T.thl, total thiol.

Table 4. Relationships between PANSS and TAS, TOS, OSI, ARE, PON1, and T.thiol values in the group of patients who continued to $\mathrm{CMHC}$.

\begin{tabular}{ccccccc}
\hline & \multicolumn{2}{c}{ PANSS-Negative Subscale } & \multicolumn{2}{c}{ PANSS-Positive Subscale } & \multicolumn{2}{c}{ PANSS-Total Subscale } \\
\cline { 2 - 7 } & $\mathrm{R}$ & $\mathrm{P}$ & $\mathrm{R}$ & $\mathrm{P}$ & $\mathrm{R}$ & $\mathrm{P}$ \\
\hline TAS & 0.168 & 0.281 & -0.088 & 0.576 & 0.122 & 0.437 \\
TOS & 0.439 & $0.003^{*}$ & 0.101 & 0.518 & 0.230 & 0.138 \\
OSI & -0.088 & 0.576 & 0.106 & 0.498 & -0.085 & 0.588 \\
ARE & 0.120 & 0.443 & 0.228 & 0.142 & 0.075 & 0.632 \\
PON1 & -0.178 & 0.254 & 0.053 & 0.734 & -0.145 & 0.352 \\
T.thl & -0.072 & 0.646 & -0.073 & 0.642 & -0.049 & 0.754 \\
\hline
\end{tabular}

${ }^{*}$ Correlation is significant at the 0.05 level (2-tailed). Pearson and Spearman correlation analyses were used. TAS, total antioxidant status; TOS, total oxidative status; OSI, oxidative stress index; PANSS, Positive and Negative Syndrome Scale; PON1, paraoxonase; ARE, arylesterase; T.thl, total thiol.

\section{Discussions}

It is known that the oxidative stress is effected from exercise and daily social activities [14]. In the literature, despite the studies about oxidative stress in patients with schizophrenia, up to now there has been no study assessing the 
relationship of oxidative stress between between the groups of patients who continued to CMHC and who did not continue to CMHC. From this point, our study is the first one. CMHC have important missions for following the daily activities, socialization and the treatment of the schizophrenia patients. In our study, ARE and T.thl levels were significantly higher and the TOS levels were found to be significantly lower in the group of patients who regularly continued to CMHC, compared to the group of patients who did not continue to CMHC. In the literature, it is seen that the schizophrenic patients were compared to healthy control subjects.

In the literature, there are inconsistent data about the schizophrenic patients' oxidant-antioxidant mechanisms [20] [21]. In a study, done with 50 schizophrenic patients, no significant difference was found at TAS, TOS, and OSI values, compared to the control group [22]. In a different study, with 64 schizophrenia patients, TAS and TOS levels were found significantly higher than controls, and T.thl levels were significantly low, and found no significant correlation with PON1 levels compared to healthy controls [20]. In another study, which 26 patients were in remission, and with 38 active schizophrenia patients, TOS and OSI levels were found to be significantly higher compared to 80 healthy control subjects, and no correlation was found at TAS levels compared to controls [23]. In a study, ARE levels were significantly higher and there was no significant difference at PON1 levels compared to control group with 41 schizophrenic patients [21]. In these studies, there is no information about the daily activities of patients.

Sistein takes place in one of the protein defence mechanisms of the body. It plays an important role at preventing the oxidative damage on cells by its functional thiol group. Protecting of cell function against the oxidative damage is done by low molecular weighted thiol and sistein depots [10]. In the literature, there is only one study finding the T.thiol values significantly lower in patients with schizophrenia compared to control group [20] [22]. In our study, we found that the schizophrenic patients who regularly continued to CMHC had higher T.thl levels, compared to schizophrenic patients who did not continue to CMHC, so we think that the CMHC may have antioxidant effects on patients. According to our findings, we can say that CMHC may reduce the oxidative stress of the schizophrenic patients by regular follow-up, by reducing the number and the frequency of psychotic attacks and by the integration of patients into society with a multidisciplinary approach.

In our study, in the group of patients who did not continue to CMHC, PANSS-Negative scores and PANSS-Total scores, showing the psychopathology of schizophrenia, were found to be significantly higher compared to the group of patients who regularly continued. We found positive significant relationship between PANSS-Negative scores and TOS, which is pointing out the relation of oxidative stress with the disease symptoms. The results of our study emphasize the importance of CMHC in the treatment of schizophrenia. 
Limitation of our study is not comparing the both patient groups to healthy control subjects. Meaningfulness of the study can be improved, by increasing the number of the patients participating and by measuring more oxidant-antioxidant molecules.

As a result, oxidative stress and negative symptoms of schizophrenia in the group of patients who continued to CMHC are less than the group which regularly did not continue. These results show us that, for schizophrenia, CMHC have an important role at patient follow-up, integration into society, and the socialization of the patients. In the treatment of psychiatric disorders, instead of hospitalization, outpatient treatment strategies must be improved for better community health services.

Because of negative symptoms, schizophrenia patients generally do not get out of their houses, even their rooms, and talk to almost nobody except family members. Continuing to CMHC forces the patients to get out of the house more often and to get rid of the sedanter life style. The patients who regularly continue to CMHC wake up early in the morning and leave their houses and join to the different activities. Because of more active life style, it can be explained that the oxidative stress is lower in the group of patients who regularly continued to CMHC.

\section{References}

[1] Angermeyer, M.C., Kühn, L. and Goldstein, J.M. (1990) Gender and the Course of Schizophrenia: Differences in Treated Outcomes. Schizophrenia Bulletin, 16, 293-307. https://doi.org/10.1093/schbul/16.2.293

[2] Salem, E.J. and Kring, M.A. (1998) The Role of Gender Differences in the Reduction of Etiologic Heterogeneity in Schizophrenia. Clinical Psychology Review, 18, 795-819. https://doi.org/10.1016/S0272-7358(98)00008-7

[3] Bassuk, E.L. and Gerson, S. (1978) Deinstitutionalization and Mental Health Services. Scientific American, 238, 46-53. https://doi.org/10.1038/scientificamerican0278-46

[4] Sağlık Bakanlığı, T.C. (2011) Toplum Ruh Sağlığı Merkezleri Hakkında Yönerge.

[5] Saugstad, F.L. (1998) Social Class, Marriage, and Fertility in Schizophrenia. Schizophrenia Bulletin, 15, 9-43.

[6] Rudyard, N. (1997) Stages in Realizing the International Diffusion of a Single Way of Working: The Clubhouse Model. New Directions for Mental Health Services, 74, 53-66.

[7] Bitanihirwe, B.K. and Woo, T.U. (2011) Oxidative Stress in Schizophrenia: An Integrated Approach. Neuroscience and Biobehavioral Reviews, 35, 878-893. https://doi.org/10.1016/j.neubiorev.2010.10.008

[8] Erel, O. (2004) A Novel Automated Direct Measurement Method for Total Antioxidant Capacity Using a New Generation, More Stable ABTS Radical Cation. Clinical Biochemistry, 37, 277-285. https://doi.org/10.1016/j.clinbiochem.2003.11.015

[9] Erel, O. (2005) A New Automated Colorimetric Method for Measuring Total Oxidant Status. Clinical Biochemistry, 38, 1103-1111. https://doi.org/10.1016/j.clinbiochem.2005.08.008 
[10] Haagen, L. and Brock, A.A. (1992) New Automated Method for Phenotyping Arylesterase (E.C.3.1.1.2.) Based upon Inhibition of Enzymatic Hydrolisis of 4-Nitrophenyl acetate. European Journal of Clinical Chemistry and Clinical Biochemistry, 30, 391-395.

[11] Eckerson, H.W., Wyte, M.C. and La Du, B.N. (1983) The Human Serum Paraoxonase/Arylesterase Polymorphism. American Journal of Human Genetics, 35, 1126-1138.

[12] Wlodek, L. (2002) Beneficial and Harmful Effects of Thiols. Polish Journal of Pharmacology, 54, 215-223.

[13] Goswami, B., Tayal, D., Gupta, N. and Mallika, V. (2009) Paraoxonase: A Multifaceted Biomolecule. Clinica Chimica Acta, 410, 1-12. https://doi.org/10.1016/j.cca.2009.09.025

[14] Powers, S.K. and Hogan, M.C. (2016) Exercise and Oxidative Stress. The Journal of Physiology, 594, 5079-5080. https://doi.org/10.1113/JP272255

[15] First, M.B. and Pincus, H.A. (2002) The DSM-IV Text Revision: Rationale and Potential Impact on Clinical Practice. Psychiatric Services, 53, 288-292. https://doi.org/10.1176/appi.ps.53.3.288

[16] Kay, S.R., Fiszbein, A. and Opler, L.A. (1987) The Positive and Negative Syndrome Scale (PANSS) for Schizophrenia. Schizophrenia Bulletin, 13, 261-275. https://doi.org/10.1093/schbul/13.2.261

[17] Harma, M., Harma, M. and Erel, O. (2003) Increased Oxidative Stres in Patients with Hydatidiform Mole. Swiss Medical Weekly, 133, 563-566.

[18] Ellman, G.L. (1959) Tissue Sulfhydryl Groups. Archives of Biochemistry and Biophysics, 82, 70-77. https://doi.org/10.1016/0003-9861(59)90090-6

[19] Hu, M.L. (1994) Measurement of Protein Thiol Groups and Glutathione in Plasma. Methods in Enzymology, 233, 380-385. https://doi.org/10.1016/S0076-6879(94)33044-1

[20] Kulaksizoglu, B. and Kulaksizoglu, S. (2016) Relationship between Neutrophil/Lymphocyte Ratio with Oxidative Stress and Psychopathology in Patients with Schizophrenia. Neuropsychiatric Disease and Treatment, 12, 1999. https://doi.org/10.2147/NDT.S110484

[21] Güneş, M., Camkurt, M.A., Bulut, M., Demir, S., İbiloğlu, A.O., Kaya, M.C., et al. (2016) Evaluation of Paraoxonase, Arylesterase and Malondialdehyde Levels in Schizophrenia Patients Taking Typical, Atypical and Combined Antipsychotic Treatment. Clinical Psychopharmacology and Neuroscience, 14, 345-350. https://doi.org/10.9758/cpn.2016.14.4.345

[22] Pazvantoglu, O., Selek, S., Okay, I.T., Sengul, C., Karabekiroglu, K., Dilbaz, N., et al. (2009) Oxidative Mechanisms in Schizophrenia and Their Relationship with Illness Subtype and Symptom Profile. Psychiatry and Clinical Neurosciences, 63, 693-700. https://doi.org/10.1111/j.1440-1819.2009.02015.x

[23] Copoglu, U.S., Virit, O., Kokacya, M.H., Orkmez, M., Bulbul, F., Erbagci, A.B., et al. (2015) Increased Oxidative Stress and Oxidative DNA Damage in Non-Remission Schizophrenia Patients. Psychiatry Research, 229, 200-205.

https://doi.org/10.1016/j.psychres.2015.07.036 